Please send trade news information and

illustrations to Arveen Bajaj at the BDJ, Nature

Publishing Group, The Macmillan Building,

4-6 Crinan Street, London N1 9XW.

Trade news is provided as a service to readers

using text and images from the manufacturer,

supplier or distributor and does not imply

endorsement by the $B D J$. Normal and prudent

research should be exercised before purchase or

use of any product mentioned.

\section{Reducing sensitivity}

Denshield from Blackwell Supplies is designed to reduce sensitivity and treat the source of the problem. It actively reduces open dentine tubules and helps to prevent exposure of the pulp nerve to stimuli both before and after bleaching or other treatments.

Denshield contains NovaMin (calcium sodium phosphosilicate), a compound composed of elements found naturally in the body, and is applied directly to the sensitive areas to completely seal any open tubules and deliver immediate short-term relief. Patients can themselves then continue the treatment for the long-term, active protection of the tooth nerve.

Reader response number 51

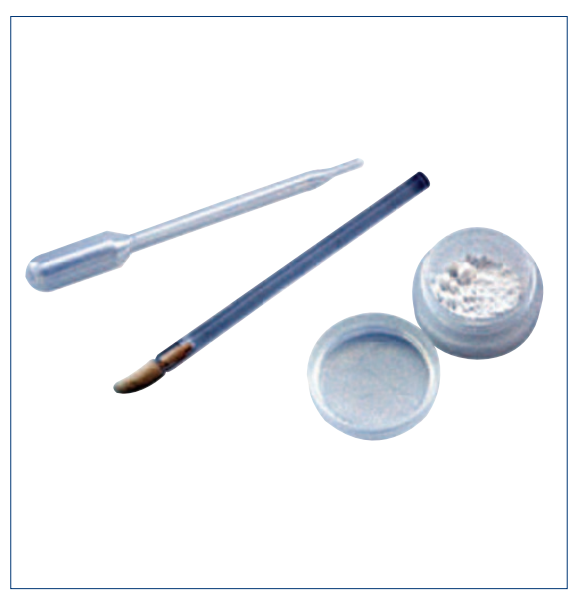

\section{New look website}

Denplan has revealed the new look for its website with the launch of its new patient site 'MyDenplan'. It allows patients access to all the latest information about its products and services at the click of a button. The new site aims to be more intuitive, simpler to read and visually more impactful, with a cleaner more modern feel.

One of the new elements to the site is the 'Members area'. This part of the site allows existing Denplan patients to login to a secure area with their own personal ID and update their contact details, for

\section{Non-eugenol temporary cement}

NexTemp from Premier is a resin based cement and does not contain either eugenol or zinc oxide, making it suitable for all temporary situations be they long or short term.

The chlorhexidine component of NexTemp acts as an antibacterial agent and the potassium nitrate part is a known desensitiser that sedates the pulp. The combination of these ingredients ensures that your temporary restorations will stay in place for as long as you need them.

Supplied in a controlled automix presentation to minimise waste, the chemically cured product goes through two phases during the setting period.

\section{Self fit mouthguard range launched}

Opro, supplier of premium, custom-fitting mouthguards is set to launch a range of self-fit mouthguards - Oproshield - which will be available in the first quarter of 2007.

The Oproshield range will comprise three levels: bronze, silver and gold and is designed to provide wearers with a wider choice of low to medium cost quality mouthguards which recognises the realities of personal budgets and

example change of address or phone numbers.

Other new features of the site include an Insurance and Claims section with information on the claims process, claim forms, how to's, policy documents and questions page, a QCtA section incorporating useful information and frequently asked questions, including information on what to do if you are moving house and Product Information which allows patients to download and print out copies of Denplan's brochures, policy documents etc.

Reader response number 53

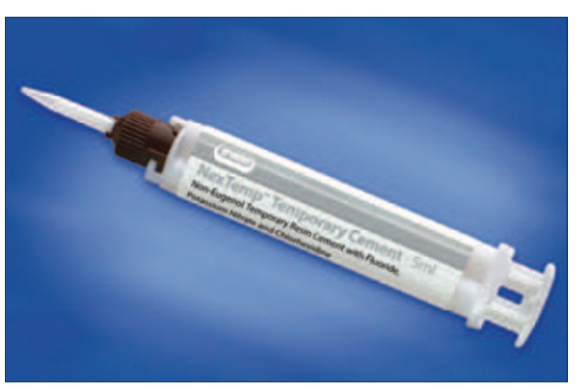

The first 'rubbery' phase allows you to remove the excess material prior to the second and final hardening of the material and it will not shine through. It is available from Myerson.

Reader response number 50

perceived values. Prices will range from $£ 1.99$ for a Bronze level mouthguard (clear) to £12.99 for a premium Gold level mouthguard (white with gold fins.)

Reader response number 52
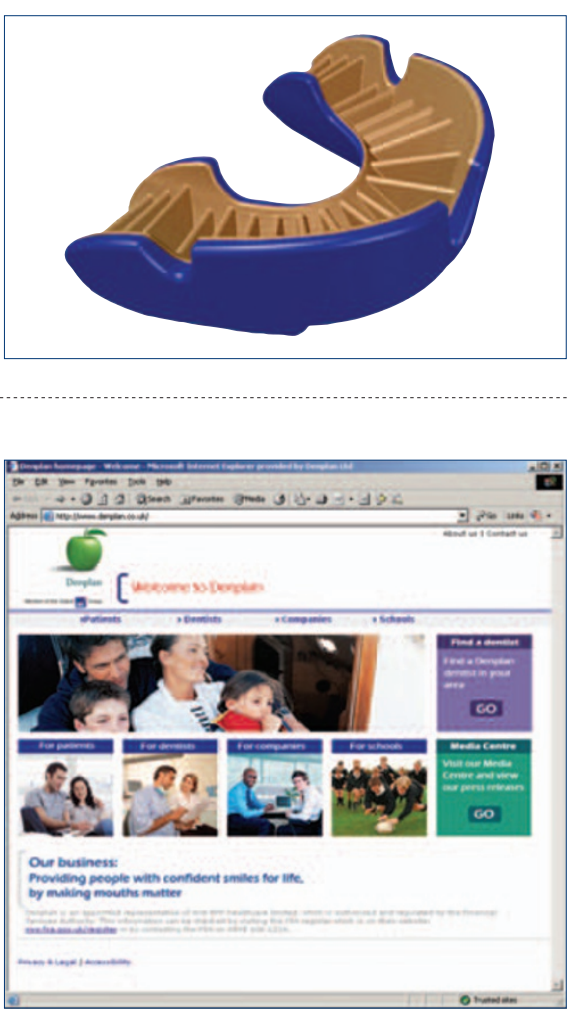

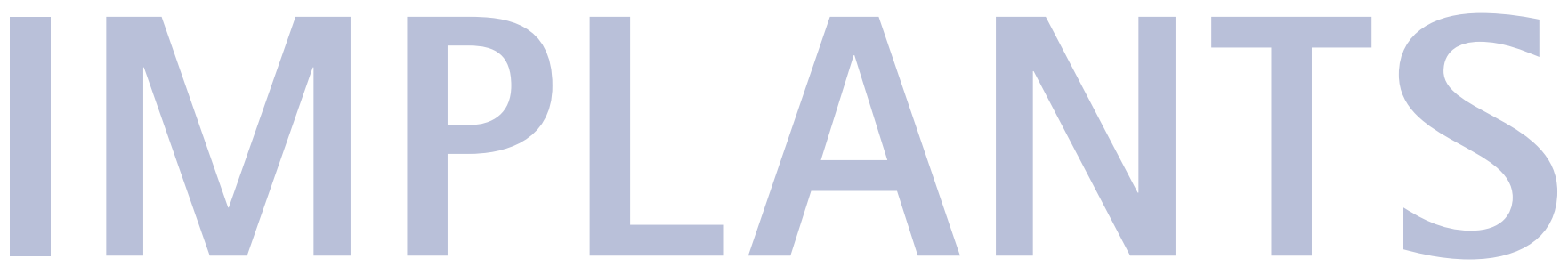

\section{Conical connection}

Ankylos from Dentsply Friadent has a precision engineered conical connection between an Ankylos abutment and the associated implant to ensure an exact fit. Literally eliminating the microgap between the implant and the abutment prevents microbial leakage and micromovement. The avoidance of both microbial and mechanical irritation reduces the risk of inflammation, thereby enhancing recovery of soft tissue and underlying bone, the company says.

Additional features of the implant system include short treatment times, one abutment fits all implant sizes, progressive thread for high primary stability and surface microstructure favours osseointegration.

Reader response number 55

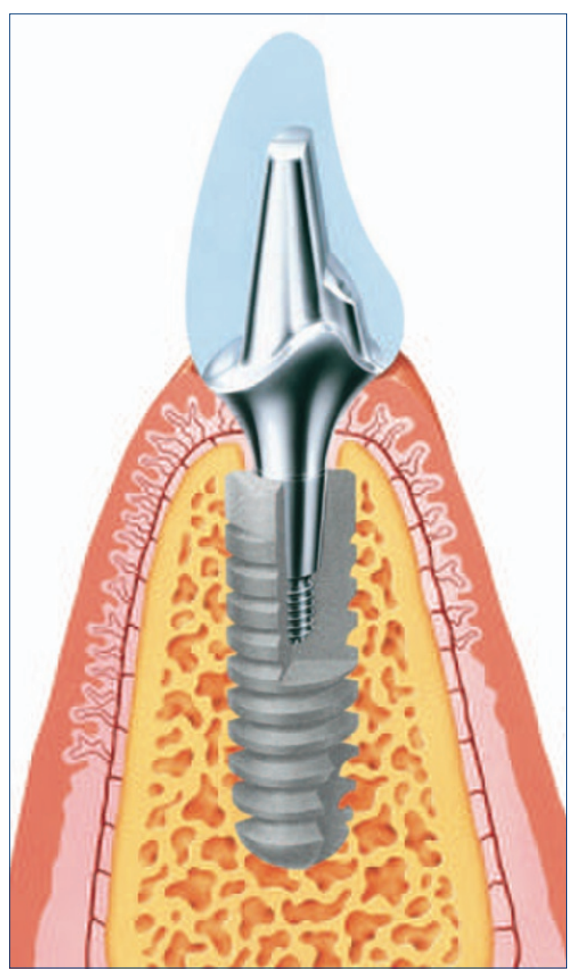

\section{Clinical flexibility}

Trycare Dental Linkline believes that the Lifecore Prima Implant System offers unprecedented clinical flexibility in a single implant system. The range includes one or two-piece implants, a streamlined surgical kit and a range of restorative options.

PrimaSolo, the one-piece tapered implant, has an integrated gold coloured abutment for optimised aesthetics through the gingiva.

PrimaConnex are two-piece tapered or straight implants featuring a unique six-lobed internal connection. They support screw-retained, cement-retained or overdenture restorations. To help enhance gingival aesthetics, the abutments are gold coloured and all the components have an anatomical design and consistent flare. Both designs feature a $1 \mathrm{~mm}$ smooth gingival collar and Lifecore's tried and tested RBM surface technology, which substantially increases bone-to-implant contact.

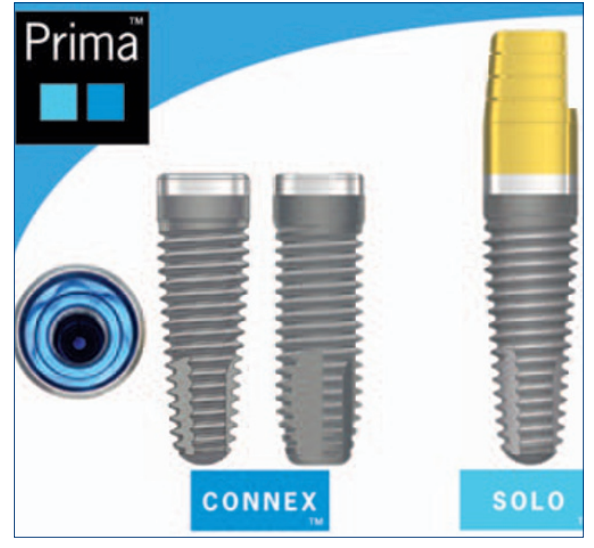

Their threads are designed for reduced insertion torque to minimise trauma and maintain stability. Prima implants are designed for immediate placement - which saves time, helps maintain soft and hard tissues, and maximises long-term aesthetic results. Designed for immediate provisionalisation, they eliminate the need for second-stage surgery and increase patient satisfaction. Reader response number 54

\section{Simplified bone collection}

Developed to maximise bone collection as part of the standard suction process, the Autografter from Trycare Dental Linkline, enables surgeons to collect autogenous graft material from any surgical site with ease.

It can be inserted 'mid-line' between two sections of suction tubing or at the end of the line, with an Hvac suction tip. The large chamber and filter screen allows multiple site collections without the need to empty the basket during the surgery. The clear sides also allow you to monitor the quantity of collected material.

Once collected the graft material can be used in either its wet amorphous form or compressed within a screen basket using a titanium plunger. The latter produces a drier more compressed pellet of autogenous graft material which is easier to handle and place.

The Ace Autografter is supplied as a kit containing the Autografter, Hvac Suction Tip, Titanium Plunger and ten Screen Assembly Baskets. All of which are also available separately.

Reader response number 56

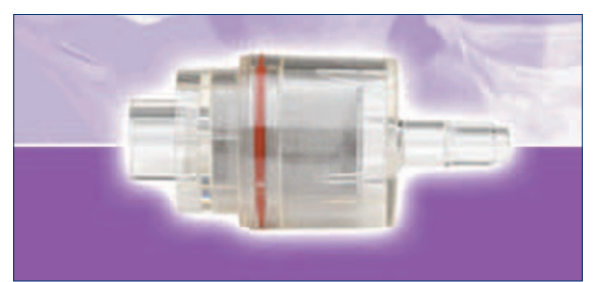




\section{See in the dark}

With GC UK's Fit Checker Black you can now see the exact fit of your full ceramic restorations prior to fitting. Fit Checker Black is a C-silicone, which is mixed quickly and easily providing you with a

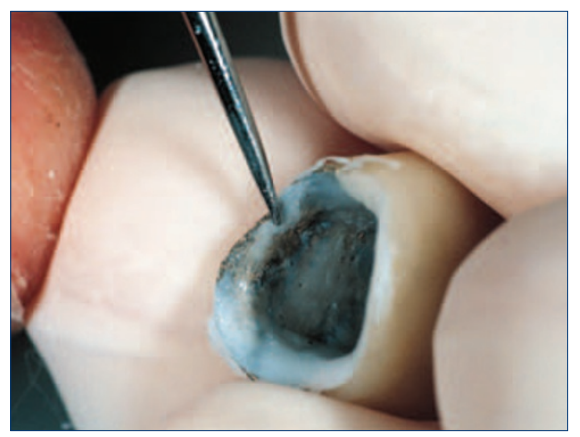

free flowing yet thixotropic diagnostic paste. The dentist can flow Fit Checker Black into the all ceramic crown or bridge, seat in the patient's mouth and after a minute the material has set. Upon removal you can see precisely how the prosthesis fits, allowing you to make final adjustments before it is too late.

Fit Checker Black peels away leaving a clean surface without the need for scrubbing your fragile crown and bridgework. The product will run alongside Fit Checker White, which can be used for checking the fit of dentures, orthodontic plates and regular crown and bridgework.

Reader response number 57

\section{Shorter treatment protocols}

Straumann's SLActive implant surface technology claims to reduce healing time to as little as three to four weeks, resulting in clear, shorter treatment protocols, increased predictability and a reduced risk with earlier loading.

Produced of pure titanium, Straumann's SLActive surface features optimal surface topography for bone cells to attach themselves. Using an innovative manufacturing process, the surface is conditioned in nitrogen and immediately preserved in an isotonic saline solution. This ensures SLActive maintains its high surface activity, which would otherwise be lost due to reaction with the atmosphere.

Reader response number 59

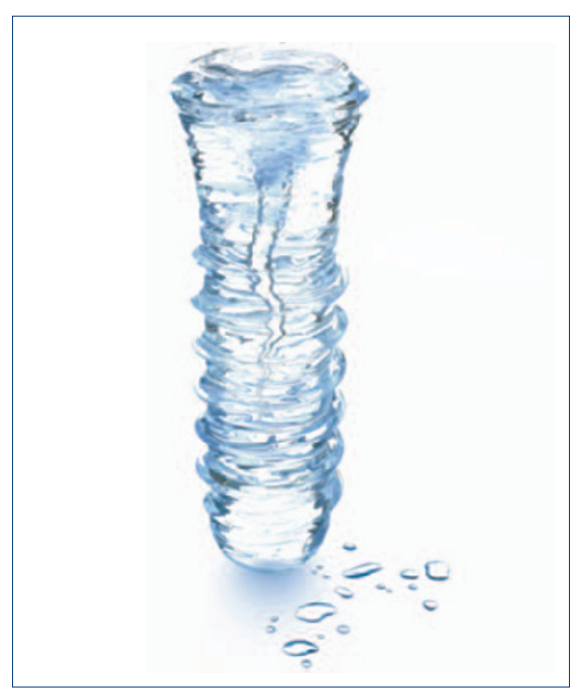

\section{Robust balanced instrument}

The Sirona T1 Classic handpieces are manufactured from high-grade titanium to provide a smooth, robust and extremely balanced instrument ideally designed for implant surgery. The fibre optic light guide provides optimum illumination with 25,000 lux output for visualising every area within the operating site.

Together with the SiroTorque L electric motor, the Sirona Classic 24:1 implant handpiece allows you to provide all necessary treatment for implantology with just one handpiece. Coolant is delivered directly to the operating site with the Kirschner/Meyer principle for optimum treatment with irrigation.

Noise levels for the Classic implant handpiece have been reduced giving less stress to user and patient whilst treatment is carried out. The smooth titanium surface is free of all potential dirt traps making the handpiece easy to repeatedly clean and sterilise in an autoclave at up to $135^{\circ} \mathrm{C}$.

Reader response number 61

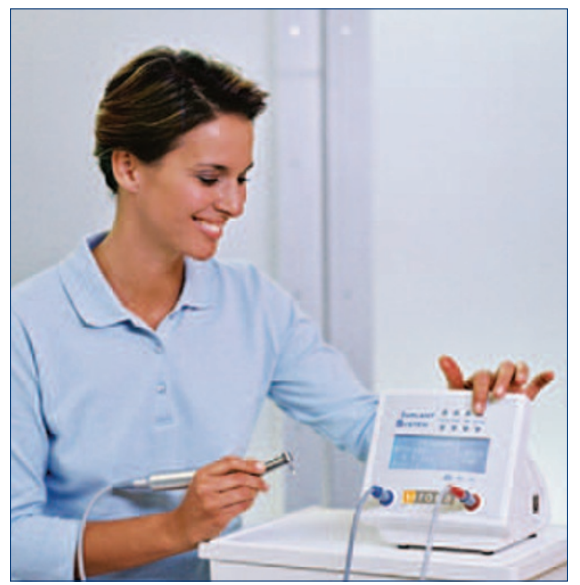

\section{Easy crown removal}

Premier Implant Cement from Myerson Limited can be used for implant retained prostheses and offers secure retention, yet permits easy removal of the crown.

The product is designed for implants but can also be used for temporaries. This non-eugenol, resin cement features high retention due to a rigid final set and low solubility, providing good marginal seal. Yet, when desired, the restoration can be easily removed due to its unique elasticity.

The automix dual-barrel syringe, with disposable mix tips, eliminates handmixing and clean-up, ensuring consistent mix and controlled dispensing. The unique two-stage auto-curing makes seating the restoration and removing excess cement quick and simple. Aesthetically shaded to match gingival tissue, this cement exhibits virtually no taste nor odour for increased patient comfort.

As an introductory offer, for a limited period, each kit contains a free Premier Implant Scaler, which can be repeatedly autoclaved and re-sharpened to safely remove excess cement and clean the abutment during prophylaxis.

Reader response number $\mathbf{5 8}$

\section{Biocompatible polymer components}

A new line of provisional components, PreFormance Posts and Temporary Cylinders have been developed by $3 \mathrm{i}$ Implant Innovations.

These components are made of a strong, biocompatible polymer material called Peek that is easily customised to a patient's tissue contours and anatomic profile.

The PreFormance Post cement-retained provisional option features the Certain QuickSeat Connection, which tells the clinician the abutment is properly seated with an audible and tactile click.

It is available in straight or $15^{\circ}$ preangled configurations for ideal angle correction, collar heights in four or six millimetres to follow gingival contours without trapping cement and Emergence Profile for more natural aesthetics.

The PreFormance Post also has a smooth surface for easy provisional restoration reline and a flat side for anti-rotation. The PreFormance Temporary Cylinder screwretained option has a knurled surface for mechanical retention of resin material and is available in hexed or non-hexed. Reader response number 60 